\title{
On the stationary motion of a self-gravitating toroidal stratum
}

\section{Giorgio Fusco}

Dipartimento di Matematica Pura ed Applicata, Università degli Studi dell'Aquila Via Vetoio, 67010 Coppito, L'Aquila, Italy

E-mail address: fusco@univaq.it

\section{Piero Negrini}

Dipartimento di Matematica, Sapienza, Università di Roma

Piazzale Aldo Moro 5, 00185 Rome, Italy

E-mail address: negrini@mat.uniroma1.it

\section{Waldyr M. Oliva}

CAMGSD and ISR, Instituto Superior Técnico, UTL - Lisboa

E-mail address: wamoliva@math.ist.utl.pt

\begin{abstract}
We consider an incompressible fluid contained in a toroidal stratum and subject only to Newtonian self-attraction. Under the assumption of infinitesimal thickness of the stratum we show the existence of stationary motions during which the stratum is approximatly a round torus (with radii $r, R$ and $R>>r$ ) that rotates around its axis and at the same time rolls on itself. Therefore each particle of the stratum describes an helix-like trajectory around the circumference of radius $R$.
\end{abstract}

\section{Introduction}

The problem of the determination of the figures of equilibrium of a selfgravitating rotating mass has received a lot of attention in the classical literature beginning with the work of Newton on the oblateness of the earth [2],[1] and [3]. The method of canals introduced by Newton was exploited by Maclaurin and Jacobi who discovered several families of ellipsoids of equilibrium. The search for stationary motions in ellipsoidal regions, including ellipsoids of equilibrium, of self-gravitating incompressible fluids was completed by Riemann [4] who developed ideas of Dirichlet [5]. Poincaré [6] adopted a global point of view and introduced the concept of bifurcation 




FiguRE 1. The geometry of $\mathcal{T}_{R}$.

in the attempt of describing the whole set of figures of equilibrium of rotating self-gravitating masses. More recently several papers have appeared approaching this classical problem with variational techniques [8] [9] [10]. The case of a rotating solid torus $\mathcal{T}$ was first considered by Poincaré [6], see also [7], and revisited in [8]. It is natural to expect that the motion studied in these papers belongs to a family of stationary motions where, beside rotating around its axis, the torus rolls on itself so that the fluid particles describe a helix-like path around the circumference $\mathcal{C}$ of radius $R$ that connects the centers of the cross sections of the torus. We can also conjecture that beside this class of stationary motions of solid self-gravitating torii, there is also the possibilty of similar stationary motions of self-gravitating toroidal strata. This last class of motions however will not contain as a special case the case of relative equilibrium since for a toroidal stratum the pressure cannot compensate the self attraction that tries to collapse the torus to the circumference $\mathcal{C}$.

In this note we consider the case of an incompressible self-gravitating toroidal stratum $\mathcal{T}_{R}$ of very small (infinitesimal) thickness. We represent $\mathcal{T}_{R}$ in the form,

see Figure 1,

$$
\left\{\begin{aligned}
\mathcal{T}_{R}= & \left\{x \in \mathcal{R}^{3}: x=R \epsilon_{1}(\phi)+(r(\theta)+\lambda) \epsilon_{r}(\theta, \phi)\right. \\
& \left.(\theta, \phi) \in S^{1} \times S^{1}, \lambda \in\left[-\frac{s(\theta)}{2}, \frac{s(\theta)}{2}\right]\right\} \\
\epsilon_{1}(\phi)= & \cos \phi e_{1}+\sin \phi e_{2} \\
\epsilon_{r}(\theta, \phi)= & \cos \theta \epsilon_{1}(\phi)+\sin \theta e_{3}
\end{aligned}\right.
$$


where $e_{j}, j=1,2,3$ are the vector of the standard basis of $\mathbb{R}^{3}, \theta \rightarrow r(\theta)$ is the polar representation of the cross section of the middle fiber $\mathcal{T}_{R}^{0}=$ $\left\{x \in \mathcal{T}_{R}: \lambda=0\right\}$ of $\mathcal{T}_{R}$ and $s(\theta)$ is the thickness of the stratum. We let $r_{0}$ the average of $r(\theta)$ and consider the case where $\varepsilon:=\frac{r_{0}}{R}<<1$. Under this assumption we regard the stationary motion of the toroidal stratum as a perturbation of the limit helicoidal motion of a cylindrical stratum that we can ideally associate to $\varepsilon=0$. Since cylinder and torus are different topological objects, the problem of continuing the motion of the fluid in the cylindrical stratum into a stationary motion in the toroidal stratum $\mathcal{T}_{R}$ is a singular perturbation problem.

Set

$$
X(\theta, \phi)=R \epsilon_{1}(\phi)+r(\theta) \epsilon_{r}(\theta, \phi) .
$$

Under the standing assumption of infinitesimal thickness the velocity field $v=v(\theta, \phi, \lambda)$ on $\mathcal{T}_{R}$ does not depend on $\lambda$ and can be computed on the middle fiber $\mathcal{T}_{R}^{0}=\left\{x=X(\theta, \phi),(\theta, \phi) \in S^{1} \times S^{1}\right\}$. Therefore we have $v=X_{\theta} \dot{\theta}+X_{\phi} \dot{\phi}$ where subscripts denote partial differentiation and time differentiation. Since we look for stationary motions which are invariant under rotations around the symmetry axis of $\mathcal{T}_{R}$ we have

$$
\left\{\begin{array}{l}
\dot{\theta}=\omega(\theta) \\
\dot{\phi}=\Omega(\theta)
\end{array}\right.
$$

for some $2 \pi$-periodic functions $\Omega, \omega$ and therefore a routine computation shows that velocity and acceleration vector fields on $\mathcal{T}_{R}^{0}$ are given by

$$
v(\theta, \phi)=\omega\left(r^{\prime} \epsilon_{r}+r \epsilon_{\theta}\right)+\Omega(R+r \cos \theta) \epsilon_{2},
$$

and

$$
\begin{aligned}
a(\theta, \phi) & =\left[\left(r^{\prime \prime}-r\right) \omega^{2}+r^{\prime} \omega^{\prime} \omega-(R+r \cos \theta) \cos \theta \Omega^{2}\right] \epsilon_{r} \\
& +\left[((R+r \cos \theta) \Omega)^{\prime} \omega+(r \cos \theta)^{\prime} \omega \Omega\right] \epsilon_{2} \\
& +\left[2 r^{\prime} \omega^{2}+r \omega^{\prime} \omega+(R+r \cos \theta) \sin \theta \Omega^{2}\right] \epsilon_{\theta},
\end{aligned}
$$

where $\epsilon_{r}$ is defined in (1.1) and $\epsilon_{\theta}=-\sin \theta \epsilon_{1}+\cos \theta e_{3}, \epsilon_{2}=-\sin \phi e_{1}+$ $\cos \phi e_{2}$. Let $n=n(\theta, \phi)=\frac{X_{\phi} \wedge X_{\theta}}{\left|X_{\phi} \wedge X_{\theta}\right|}$ the exterior unit normal to $\mathcal{T}_{R}^{0}$ at $X(\theta, \phi)$ and $\delta=\delta(\theta)$ the thickness of the stratum along $n$ that is $\delta=s n \cdot \epsilon_{r}$.

The balance between inertial forces and newtonian self-attraction at the typical point $X(\phi, \theta)$ of $\mathcal{T}_{R}^{0}$ reads

$$
a(\phi, \theta)=G \mu \int_{S^{1} \times S^{1}}^{*} \frac{X(\alpha, \beta)-X(\theta, \phi)}{|X(\alpha, \beta)-X(\theta, \phi)|^{3}}\left|X_{\phi} \wedge X_{\theta}\right|(\alpha, \beta) \delta(\alpha) d \alpha d \beta(, 1.6)
$$

where $\mu$ is the constant density of the fluid, $G$ the gravitational constant and the integral in the r.h.s. is to be intended in the sense of Cauchy 
principal value. That is

$$
\int_{S^{1} \times S^{1}}^{*}=\lim _{l \rightarrow 0^{+}} \int_{\left(S^{1} \times S^{1}\right) \backslash B_{l}}
$$

with $B_{l}$ a ball or radius $l$ centered at $X(\phi, \theta)$.

Due to the axial symmetry of the motion and of the mass distribution in $\mathcal{T}_{R}$ the component on $\epsilon_{2}$ of the r.h.s. of (1.6) vanishes and therefore the equation of motion (1.6) admits the first integral

$$
(R+r \cos \theta)^{2} \Omega=J(R),
$$

that expresses the conservation of momentum of momentum with respect to the symmetry axis of $\mathcal{T}_{R}$. Equation (1.6) must be complemented with the continuity equation that states the constance of the flux through the section $S_{\theta}$ of $\mathcal{T}_{R}$ obtained by cutting $\mathcal{T}_{R}$ normally to $\mathcal{T}_{R}^{0}$ along the line $\theta=$ const :

$$
\left|S_{\theta}\right| v \cdot \epsilon_{t}=C(R) .
$$

where $\epsilon_{t}=n \wedge \epsilon_{2}$ is a unit vector tangent at $X(\theta, \phi)$ to the line $\phi=$ const on $\mathcal{T}_{R}^{0}$. Observing that

$$
\left\{\begin{aligned}
\epsilon_{t} & =\frac{\left(r \epsilon_{r}\right)_{\theta}}{\left|\left(r \epsilon_{r}\right)_{\theta}\right|}=\frac{r^{\prime} \epsilon_{r}+r \epsilon_{\theta}}{\sqrt{r^{\prime 2}+r^{2}}}, \\
n & =\frac{r \epsilon_{r}-r^{\prime} \epsilon_{\theta}}{\sqrt{r^{\prime 2}+r^{2}}}, \\
\delta & =\frac{s r}{\sqrt{r^{\prime 2}+r^{2}}}, \\
\left|S_{\theta}\right| & =2 \pi(R+r \cos \theta) \delta,
\end{aligned}\right.
$$

where ' denotes differentiation with respect to $\theta$, we can rewrite (1.8) in the explicit form

$$
(R+r \cos \theta) s r \omega=C(R) .
$$

By means of the first integral (1.7) and the continuity equation (1.10) we can determine $s=s(\theta)$ and $\Omega=\Omega(\theta)$ once $r=r(\theta)$ and $\omega=\omega(\theta)$ are known. This allows for transforming (1.6) and (1.10) into an equivalent system, see (2.7) below, for the unknowns $r=r(\theta), \omega=\omega(\theta)$. We let $r_{0}$ and $\omega_{0}$ be the averages of $r$ and $\omega$ and we represent the unknowns $r$ and $\omega$ in the form

$$
\begin{gathered}
r=r_{0}(1+\varepsilon \rho), \\
\omega=\omega_{0}(1+\varepsilon w),
\end{gathered}
$$

where $\varepsilon:=\frac{r_{0}}{R}$ is regarded as a small parameter and $\rho$ and $w$ are $2 \pi$-periodic functions with zero average. We observe that the representation of $\mathcal{T}_{R}$ in (1.1) is not unique. To make the representation (1.1) of $\mathcal{T}_{R}$ unique we impose on the unknown $\rho$ the conditions 


$$
\left\{\begin{array}{l}
\int_{S^{1}} \rho \cos \theta=0 \\
\int_{S^{1}} \rho \sin \theta=0
\end{array}\right.
$$

In the following we sketch the proof of the existence, for small $\varepsilon>0$, of a solution $r, \omega, s, \Omega$ of the integral-differential equation (1.6). This is stated in the following theorem. For a complete detailed proof we refer the reader to the forthcoming paper [11].

Theorem 1.1. Given $r_{0}>0$ and $\omega_{0}>0$, there exists $\varepsilon_{0}>0$ such that for each $\varepsilon=\frac{r_{0}}{R}<\varepsilon_{0}$ the system (2.7), has a $2 \pi$-periodic solution $r=$ $r_{0}(1+\varepsilon \rho), \omega=\omega_{0}(1+\varepsilon w)$ such that:

(i): The maps $\rho$ and $w$ are of class $C^{2, \gamma}$ and $C^{1, \gamma}$ respectively for some $\gamma \in(0,1)$. Moreover $\rho$ and $w$ have zero average and $\rho$ satisfies (1.12).

(ii): $\rho$ and $w$ satisfy the estimates

$$
\begin{array}{r}
\lim _{\varepsilon \rightarrow 0^{+}}\|\rho\|_{W^{2,2}}=0, \\
\lim _{\varepsilon \rightarrow 0^{+}}\|w-\bar{w}\|_{W^{1,2}}=0,
\end{array}
$$

where $\bar{w}:=-\frac{1}{4} \cos \theta$.

(iii): The solution is unique in the set of maps that satisfy:

$$
\|\rho\|_{W^{2,2}}+\|w\|_{W^{1,2}} \leq 2\|\bar{w}\|_{W^{1,2}} \text {. }
$$

(iv): The function $s$ is of class $C^{1, \gamma}$ and the function $\Omega$ is of class $C^{2, \gamma}$. Moreover

$$
\begin{aligned}
\|s-\bar{s}\|_{W^{1,2}} & =o(\varepsilon), \\
\|\Omega-\bar{\Omega}\|_{W^{2,2}} & =o(\varepsilon),
\end{aligned}
$$

where $\bar{s}=\frac{\omega_{0}^{2} r_{0}}{2 \pi \mu G}\left(1-\varepsilon \frac{3}{4} \cos \theta\right)$ and $\bar{\Omega}= \pm \omega_{0} \varepsilon \nu(\varepsilon)$ with $\nu(\varepsilon)$ such that

$$
c \leq \frac{\nu(\varepsilon)}{\sqrt{1+\log \frac{1}{\varepsilon}}} \leq C,
$$

for some $c, C>0$ independent from $\varepsilon$.

From Theorem 1.1 we see that, during the stationary motion of the stratum, the cross section of the torus remains nearly circular: the error $r_{0} \varepsilon \rho$ is a $o(\varepsilon)$, the angular velocity $\omega$ differes from its average by a term of $O(\varepsilon)$ and it is smaller than the average at $\theta=0$ and larger than $\omega_{0}$ at $\theta=\pi$. The thickness $s$ of the stratum is smaller at $\theta=0$ and larger at $\theta=\pi$. The angular velocity $\Omega$ around the axis of the torus is almost independent of $\theta$. 
The paper is organized as follows. In sec. 2 we reduce (1.6) to a system, see (2.7), of two scalar equation for the unknowns $r, \omega$ by eliminating $s$ and $\Omega$ via the continuity equation (1.10) and the first integral (1.7). In sec. 3 we present explicit expressions of the significant part of the newtonian forces and study their dependence on the parameter $\varepsilon$. Using the analysis in sec. 3 we write, in sec. 4 , the system for $r, \omega$ as a weak nonlinear equation which, for $\varepsilon<<1$, can be solved leading to the proof of Theorem 1.1.

We denote by $\varphi_{0}, \varphi_{n}^{j}, j=1,2, n=1, \cdots$ the Fourier coefficient of a $2 \pi$-periodic integrable function $\varphi$.

\section{The system for $r$ and $\omega$}

In the following, if $h: \mathcal{R} \rightarrow \mathcal{R}$ is a $2 \pi$-periodic function, we set

$$
\langle h\rangle=\int_{S^{1}} h(\theta) d \theta .
$$

Let $F$ the integral term on the r.h.s. of (1.6), that is the force of newtonian interaction. Define

$$
f^{r}=F \cdot \epsilon_{r}, \quad f^{\theta}=F \cdot \epsilon_{\theta}, \quad f=F \cdot \epsilon_{1}=f^{r} \cos \theta-f^{\theta} \sin \theta .
$$

The axial symmetry of the problem implies that, as can be also verified by inspecting the expression of $F$, the components $f^{r}, f^{\theta}, f$ depend only on the variable $\theta$. Rewrite (1.6) in the form

$$
v_{\theta} \omega+v_{\phi} \Omega=F .
$$

From the kinematic identity

$$
v_{\theta} \cdot \epsilon_{1}=\left(v \cdot \epsilon_{1}\right)_{\theta} \quad \Rightarrow\left\langle\left(v \cdot \epsilon_{1}\right)_{\theta}\right\rangle=0,
$$

and (2.3) it follows

$$
\left\langle v_{\phi} \cdot \epsilon_{1} \frac{\Omega}{\omega}\right\rangle=-\left\langle(R+r \cos (\cdot)) \frac{\Omega^{2}}{\omega}\right\rangle=\left\langle\frac{f}{\omega}\right\rangle,
$$

where we have also used (1.4) that implies $v_{\phi} \cdot \epsilon_{1}=-(R+r \cos \theta) \Omega$. From (1.7) and (2.5) we obtain

$$
\left\{\begin{array}{l}
J^{2}(R)=-\frac{\left\langle\frac{f}{\omega}\right\rangle}{\left\langle\frac{1}{\omega(R+\cos (\cdot))^{3}}\right\rangle}, \\
\Omega^{2}(\theta)=-\frac{1}{(R+r \cos \theta)^{4}} \frac{\left\langle\frac{f}{\omega}\right\rangle}{\left\langle\frac{1}{\omega(R+r \cos (\cdot))^{3}}\right\rangle} .
\end{array}\right.
$$


By means of this expressions of $\Omega^{2}$ and (2.2), (1.5), from (1.6), after dividing by $\omega$, it follows

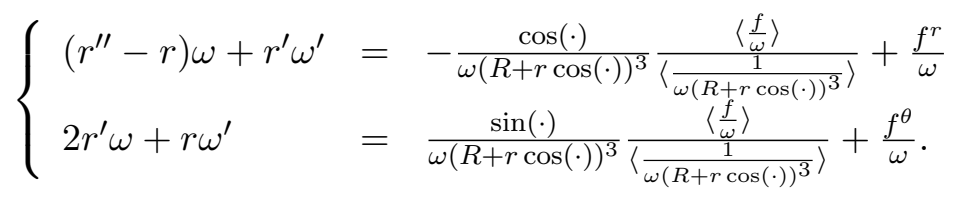

We let $\mathcal{F}^{r}$ and $\mathcal{F}^{\theta}$ the expressions on the r.h.s. of (2.7).

\section{Analysis of $\mathcal{F}^{r}$ and $\mathcal{F}^{\theta}$}

We look for a solution $(\rho, w)$ of $(2.7)$ in the space $W^{2,2}(-\pi, \pi) \times W^{1,2}(-\pi, \pi)$ and let the Fourier series of $\rho$ and $w$ be:

$$
\left\{\begin{array}{l}
\rho=\sum_{2}^{\infty}\left(\rho_{n}^{1} \cos (n \cdot)+\rho_{n}^{2} \sin (n \cdot)\right) \\
w=\sum_{1}^{\infty}\left(w_{n}^{1} \cos (n \cdot)+w_{n}^{2} \sin (n \cdot)\right)
\end{array}\right.
$$

where we have kept into account that $\rho$ and $w$ have zero average together with (1.12).

We set $\|(\rho, w)\|:=\|\rho\|_{W^{2,2}}+\|w\|_{W^{1,2}}$ and assume

$$
\|(\rho, w)\| \leq M
$$

for some constant $M>0$ to be chosen later.

A basic step in the proof of Theorem 1.1 is the the determination of the main terms in $\varepsilon$ of $\mathcal{F}^{r}$ and $\mathcal{F}^{\theta}$ and the analysis of their dependence from the Fourier coefficients of $\rho$ and $w$. From (1.2), (1.9) and (1.10) we obtain

$$
\begin{aligned}
\delta\left|X_{\theta} \wedge X \phi\right| & =\frac{r s}{\sqrt{r^{2}+r^{2}}} \sqrt{r^{2}+r^{2}}(R+r \cos (\cdot)) \\
& =r s(R+r \cos (\cdot))=C(R) \frac{1}{\omega} .
\end{aligned}
$$

We note that from (3.3), we have

$$
\int_{S^{1} \times S^{1}} h \delta\left|X_{\theta} \wedge X \phi\right| d \theta d \phi=C(R) \int_{S^{1}}\left\langle\frac{h}{\omega}\right\rangle d \phi,
$$

for each function $h: S^{1} \times S^{1} \rightarrow \mathcal{R}$. Since $f^{r}, f^{\theta}, f$ depend only on the variable $\theta$ it suffices to compute these components at $\phi=0$. From this observation and (3.3) it follows

$$
\left\{\begin{array}{l}
f^{r}=\frac{G \mu C(R)}{R^{2}} \int_{S^{1} \times S^{1}} \frac{1}{\omega(\alpha)} \frac{N^{r}(\alpha, \beta, \theta)}{D^{3}(\alpha, \beta, \theta)} d \alpha d \beta, \\
f^{\theta}=\frac{G \mu C(R)}{R^{2}} \int_{S^{1} \times S^{1}} \frac{1}{\omega(\alpha)} \frac{N^{\theta}(\alpha, \beta, \theta)}{D^{3}(\alpha, \beta, \theta)} d \alpha d \beta,
\end{array}\right.
$$


where, after setting $\varepsilon=\frac{r_{0}}{R}$ and

$$
z(t)=2(1-\cos t)
$$

it results

$$
\begin{aligned}
& \left\{\begin{array}{r}
N^{r}=\frac{1}{2}(-z(\beta) \cos \theta+\varepsilon(-z(\beta) \cos \alpha \cos \theta-z(\alpha-\theta)) \\
\left.+\varepsilon^{2}(-z(\beta) \rho(\alpha) \cos \alpha \cos \theta-z(\alpha-\theta) \rho(\alpha)+2(\rho(\alpha)-\rho(\theta)))\right) \\
N^{\theta}=\frac{1}{2}(z(\beta) \sin \theta+\varepsilon(z(\beta) \cos \alpha \sin \theta+2 \sin (\alpha-\theta)) \\
\left.+\varepsilon^{2}(z(\beta) \rho(\alpha) \cos \alpha \sin \theta+2 \rho(\alpha) \sin (\alpha-\theta))\right)
\end{array}\right. \\
& \begin{aligned}
D^{2}= & z(\beta)[1+\varepsilon(\cos \alpha+\cos \theta) \\
& +\varepsilon^{2}(\cos \alpha \cos \theta+\rho(\alpha) \cos \alpha+\rho(\theta) \cos \theta) \\
& \left.+\varepsilon^{3}(\rho(\alpha)+\rho(\theta)) \cos \alpha \cos \theta+\varepsilon^{4} \rho(\alpha) \rho(\theta) \cos \alpha \cos \theta\right] \\
& +\varepsilon^{2} z(\alpha-\theta)\left[1+\varepsilon(\rho(\alpha)+\rho(\theta))+\varepsilon^{2}(\rho(\alpha) \rho(\theta)\right. \\
& \left.\left.+\frac{(\rho(\alpha)-\rho(\theta))^{2}}{z(\alpha-\theta)}\right)\right]
\end{aligned}
\end{aligned}
$$

We let $D_{0}^{2}(\beta, \alpha):=z(\beta)+\varepsilon^{2} z(\alpha)$ and define $d=d(\rho ; \alpha, \beta, \theta, \varepsilon)$ by setting

$$
D^{2}=D_{0}^{2}(\beta, \alpha-\theta)(1+d(\rho ; \alpha, \beta, \theta, \varepsilon)) .
$$

Since $d$ is a quantity of $O(\varepsilon)$ one can check that, provided $\varepsilon \in\left(0, \varepsilon_{0}\right]$, for some $\varepsilon_{0}>0$, we have

$$
\frac{1}{D^{3}}=\frac{1}{D_{0}^{3}(\beta, \alpha-\theta)}\left(1+\sum_{n=1}^{\infty}\left(\begin{array}{c}
-\frac{3}{2} \\
n
\end{array}\right) d(\rho ; \alpha, \beta, \theta, \varepsilon)^{n}\right),
$$

and the series on the r.h.s. converges absolutely and uniformly on $S^{1} \times$ $S^{1} \times S^{1}$.

Similarly

$$
\frac{1}{\omega}=\frac{1}{\omega_{0}}\left(1+\sum_{m=1}^{\infty} \epsilon^{m}\left(\begin{array}{c}
-1 \\
m
\end{array}\right) w^{m}\right)=\frac{1}{\omega_{0}}\left(1+\sum_{m=1}^{\infty}(-1)^{m} \varepsilon^{m} w^{m}\right)
$$

It follows

$$
\frac{1}{\omega D^{3}}=\frac{1}{\omega_{0} D_{0}^{3}(\beta, \alpha-\theta)}(1+S(\rho, w ; \alpha, \beta, \theta, \varepsilon)),
$$

where $S(\rho, w)=S(\rho, w ; \alpha, \beta, \theta, \varepsilon)$ is given by 


$$
S(\rho, w)=\sum_{n=1}^{\infty} \sum_{m=0}^{n}(-1)^{m} \varepsilon^{m}\left(\begin{array}{c}
-\frac{3}{2} \\
n-m
\end{array}\right) w^{m} d(\rho)^{n-m}
$$

and, under the assumption (3.2), $S(\rho, w)$ is Hölder continuous for some $\gamma \in(0,1)$ and

$$
\begin{aligned}
\|S(\rho, w)\|_{C^{0, \gamma}} & \leq C \varepsilon \\
\left\|S\left(\rho_{1}, w_{1}\right)-S\left(\rho_{2}, w_{2}\right)\right\|_{C^{0, \gamma}} & \leq C \varepsilon\left\|\left(\rho_{1}, w_{1}\right)-\left(\rho_{2}, w_{2}\right)\right\| .
\end{aligned}
$$

By means of (3.12) and some computation we can rewrite (3.5) in the form:

$$
\left\{\begin{array}{l}
f^{r}=\frac{G \mu C(R)}{2 \omega_{0} R^{2}} \sum_{h=1}^{3} \int_{S^{1} \times S^{1}} K_{h}^{r}(\beta, \alpha-\theta, \varepsilon)\left(1+S_{h}^{r}(\rho, w ; \alpha, \beta, \theta, \varepsilon)\right) d \alpha d \beta \\
f^{\theta}=\frac{G \mu C(R)}{2 \omega_{0} R^{2}} \sum_{h=1}^{2} \int_{S^{1} \times S^{1}} K_{h}^{\theta}(\beta, \alpha-\theta, \varepsilon)\left(1+S_{h}^{\theta}(\rho, w ; \alpha, \beta, \theta, \varepsilon)\right) d \alpha d \beta
\end{array}\right.
$$

where

$$
\begin{aligned}
K_{1}^{r} & =\frac{2 \varepsilon^{2}(\rho(\alpha)-\rho(\theta))}{D_{0}^{3}(\beta, \alpha-\theta)}, \\
K_{2}^{r}=\frac{1}{\varepsilon} K_{2} & =-\frac{\varepsilon z(\alpha-\theta)}{D_{0}^{3}(\beta, \alpha-\theta)}, \\
K_{3}^{r}=-\cos (\theta) K_{3} & =-\frac{z(\beta) \cos \theta}{D_{0}^{3}(\beta, \alpha-\theta)}, \\
K_{1}^{\theta}=\frac{1}{\varepsilon} K_{1} & =\frac{2 \varepsilon \sin (\alpha-\theta)}{D_{0}^{3}(\beta, \alpha-\theta)}, \\
K_{2}^{\theta}=\sin (\theta) K_{3} & =\frac{z(\beta) \sin \theta}{D_{0}^{3}(\beta, \alpha-\theta)},
\end{aligned}
$$

where $S_{h}^{r}(\rho, w)$ and $S_{h}^{\theta}(\rho, w)$ are functions similar to $S(\rho, w)$ that satisfy (3.14).

The singular operators $\mathcal{K}_{1}^{r}$ and $\mathcal{K}_{j}, j=1,2,3$ introduced above are of the type studied in [12]. The properties of these operators are listed in the following lemma that we quote from [11] without proof. 
Lemma 3.1. Let $\sigma: S^{1} \times S^{1} \times S^{1} \rightarrow \mathcal{R}$ a map of class $C^{0, \gamma}, \gamma \in(0,1)$. Then

$$
\begin{aligned}
& \mathcal{K}_{1}^{r} \sigma(\theta)=\int_{S^{1} \times S^{1}}^{*} K_{1}^{r}(\alpha, \beta, \theta, \epsilon) \sigma(\alpha, \beta, \theta) d \alpha d \beta \\
& \mathcal{K}_{j} \sigma(\theta)=\int_{S^{1} \times S^{1}}^{*} K_{j}(\alpha, \beta, \theta, \epsilon) \sigma(\alpha, \beta, \theta) d \alpha d \beta, \quad j=1,2,3
\end{aligned}
$$

define continuous linear maps $\mathcal{K}_{1}^{r}, \mathcal{K}_{j}: C^{0, \gamma} \rightarrow C^{0, \gamma^{\prime}}, j=1,2,3$ for all $0<\gamma^{\prime}<\frac{\gamma}{1+\gamma}$. Moreover:

$$
\begin{aligned}
\left\|\mathcal{K}_{1}^{r}\right\| & <C_{\gamma^{\prime}}\|\rho\|_{C^{1, \gamma}}, \\
\left\|\mathcal{K}_{1}\right\| & <C_{\gamma^{\prime}}, \\
\left\|\mathcal{K}_{2}\right\| & <C, \\
\left\|\mathcal{K}_{3}\right\| & <C\left(1+\log \frac{1}{\varepsilon}\right),
\end{aligned}
$$

where $C$ and $C_{\gamma^{\prime}}$ are constants and $\lim _{\gamma^{\prime} \rightarrow \frac{\gamma}{1+\gamma}} C_{\gamma^{\prime}}=+\infty$.

From Lemma 3.1 and the fact that $S_{h}^{r}(\rho, w)$ and $S_{h}^{\theta}(\rho, w)$ satisfy $(3.14)$ it follows that $f^{r}$ and $f^{\theta}$ are $C^{0, \gamma}$ for some $\gamma \in(0,1)$. On the other hand by an elementary but long computation it is possible to determine the Fourier coefficients of the function $\mathcal{K}_{j} \varphi, j=1,2,3$ in term of the Fourier coefficients of $\varphi$ and the same is true for the functions $\mathcal{K}_{j} \tilde{\varphi}, \mathcal{K}_{j} \hat{\varphi}, j=1,2,3$ where

$$
\tilde{\varphi}=-\frac{3}{2}\left(\frac{z(\beta)}{D_{0}^{2}(\beta, \alpha-\theta)} \varphi(\alpha) ; \quad \hat{\varphi}=-\frac{3}{2} \frac{\varepsilon^{2} z(\alpha-\theta)}{D_{0}^{2}(\beta, \alpha-\theta)} \varphi(\alpha) .\right.
$$

By utilizing this fact, after singling out the terms of $O(\varepsilon)$ in $S_{h}^{r}(\rho, w)$ and $S_{h}^{\theta}(\rho, w)$, through a standard but tedious computation one obtains the following basic result which yields explicit expressions of $\mathcal{F}^{r}$ and $\mathcal{F}^{\theta}$ in term of the Fourier coefficients of $\rho$ and $w$. We set $c(\varepsilon):=\frac{G \mu C(R)}{2 \omega_{0}^{3} r_{0}^{2} R}$ and denote by symbol $o\left(\varepsilon^{k}\right)^{0, \gamma}$ a map $h(\rho, w ; \alpha, \theta, \varepsilon)$ such that

$$
\begin{aligned}
\|h(\rho, w)\|_{C^{0, \gamma}} & \leq C(\varepsilon) \varepsilon^{k} \\
\left\|h\left(\rho_{1}, w_{1}\right)-h\left(\rho_{2}, w_{2}\right)\right\|_{C^{0, \gamma}} & \leq C(\varepsilon) \varepsilon^{k}\left\|\left(\rho_{1}, w_{1}\right)-\left(\rho_{2}, w_{2}\right)\right\|,
\end{aligned}
$$

where $C(\varepsilon)$ satisfies $\lim _{\epsilon \rightarrow 0^{+}} C(\epsilon)=0$. 
Proposition 3.1. We have

$$
\begin{aligned}
& \frac{\mathcal{F}^{r}}{\omega_{0} r_{0}}=-4 \pi c(\varepsilon)+4 \pi \varepsilon c(\varepsilon)\left[\frac{1}{2} \cos \theta\right. \\
&\left.+\sum_{2}^{\infty}(1-n)\left(\rho_{n}^{1} \cos n \theta+\rho_{n}^{2} \sin n \theta\right)+w(\theta)\right]+\varepsilon \mathcal{N}_{F}^{r} \\
& \frac{\mathcal{F}^{\theta}}{\omega_{0} r_{0}}=4 \pi \varepsilon c(\varepsilon)\left[\sum_{1}^{\infty}\left(-w_{n}^{2} \cos n \theta+w_{n}^{1} \sin n \theta\right)+\frac{1}{2} \sin \theta\right]+\varepsilon \mathcal{N}_{F}^{\theta} .
\end{aligned}
$$

where $\mathcal{N}_{F}^{r}=\mathcal{N}_{F}^{r}(\rho, w ; \theta, \varepsilon), \mathcal{N}_{F}^{\theta}=\mathcal{N}_{F}^{\theta}(\rho, w ; \theta, \varepsilon)$ are $o\left(\varepsilon^{0}\right)^{0, \gamma}$.

\section{The proof of Theorem $\mathbf{1 . 1}$}

We let $X$ the set of the pairs $(\rho, w)$ of $2 \pi$-periodic functions $\rho \in W^{2,2}(-\pi, \pi), w \in W^{1,2}(-\pi, \pi)$ that satisfy (3.2) and are of the form (3.1) that is satisfy

$$
\begin{array}{ll}
\rho_{0}=0, & w_{0}=0, \\
\rho_{1}^{j}=0, & j=1,2 .
\end{array}
$$

$X$ with the distance induced by the norm $\|(\rho, w)\|:=\|\rho\|_{W^{2,2}}+\|w\|_{W^{1,2}}$ is a complete metric space. that

We say that a map $h(\rho, w ; \theta, \varepsilon)$ that satisfies $(3.2)$ is $o_{X}\left(\varepsilon^{k}\right)$ if $h$ is such

$$
\begin{aligned}
\|h(\rho, w)\|_{L^{2}} & \leq C(\varepsilon) \varepsilon^{k}, \\
\left\|h\left(\rho_{1}, w_{1}\right)-h\left(\rho_{2}, w_{2}\right)\right\|_{L^{2}} & \leq C(\varepsilon) \varepsilon^{k}\left\|\left(\rho_{1}, w_{1}\right)-\left(\rho_{2}, w_{2}\right)\right\|,
\end{aligned}
$$

where $\lim _{\epsilon \rightarrow 0^{+}} C(\varepsilon)=0$.

Remark 4.1. If $(\rho, w) \in X$ then

$$
h(\rho, w ; \theta, \varepsilon)=o\left(\varepsilon^{k}\right)^{0, \gamma} \Rightarrow h(\rho, w ; \theta, \varepsilon)=o_{X}\left(\varepsilon^{k}\right)
$$

Let $\left(\begin{array}{c}\frac{a^{r}}{\omega} \\ \frac{a^{\theta}}{\omega}\end{array}\right)$ the l.h.s. of (2.7). Then from (3.1) we obtain

$$
\begin{aligned}
& \frac{1}{\omega_{0} r_{0}} \frac{a^{r}}{\omega}=-1+\varepsilon\left(\rho^{\prime \prime}-\rho-w\right)+\varepsilon \mathcal{N}_{a}^{r}, \\
& \frac{1}{\omega_{0} r_{0}} \frac{a^{\theta}}{\omega}=\varepsilon\left(2 \rho^{\prime}+w^{\prime}\right)+\varepsilon \mathcal{N}_{a}^{\theta} .
\end{aligned}
$$


where $\mathcal{N}_{a}^{r}=\mathcal{N}_{a}^{r}(\rho, w ; \theta, \varepsilon)$ and $\mathcal{N}_{a}^{\theta}=\mathcal{N}_{a}^{\theta}(\rho, w ; \theta, \varepsilon)$ are $o_{X}\left(\varepsilon^{0}\right)$. We are now in the position of transforming the equations (2.7) into an infinite set of equations for the Fourier coefficients of the unknowns $\rho$ and $w$.

From (4.4) and Proposition 3.1 it follows that we can rewrite (2.7) in the form

$$
\begin{aligned}
&-1+\epsilon\left(\rho^{\prime \prime}-\rho-w\right)=-4 \pi c(\epsilon)+4 \pi \epsilon c(\epsilon)\left[\frac{1}{2} \cos \theta\right. \\
&\left.+\sum_{2}^{\infty}(1-n)\left(\rho_{n}^{1} \cos n \theta+\rho_{n}^{2} \sin n \theta\right)+w(\theta)\right]+\epsilon \mathcal{N}^{r}, \\
& \epsilon\left(2 \rho^{\prime}+w^{\prime}\right)=4 \pi \epsilon c(\epsilon)\left[\sum_{1}^{\infty}\left(-w_{n}^{2} \cos n \theta+w_{n}^{1} \sin n \theta\right)+\frac{1}{2} \sin \theta\right]+\epsilon \mathcal{N}^{\theta},
\end{aligned}
$$

where we have set $\mathcal{N}^{r}=\mathcal{N}_{F}^{r}-\mathcal{N}_{a}^{r}$ and $\mathcal{N}^{\theta}=\mathcal{N}_{F}^{\theta}-\mathcal{N}_{a}^{\theta}$. From Remark 4.1 and (4.4) and Proposition 3.1 it follows that $\mathcal{N}^{r}$ and $\mathcal{N}^{\theta}$ are $o_{X}\left(\epsilon^{0}\right)$.

By projecting $(4.5)_{1}$ on the subspace of constant functions we obtain

$$
c(\epsilon)=\frac{1}{4 \pi}-\frac{1}{8 \pi} \epsilon \mathcal{N}_{0}^{r} .
$$

Using (4.6) we rewrite (4.5) in the form

$$
\begin{aligned}
\rho^{\prime \prime}-\rho-w-\left[\sum_{2}^{\infty}(1-n)\left(\rho_{n}^{1} \cos n \theta+\rho_{n}^{2} \sin n \theta\right)+w(\theta)\right] & =\frac{1}{2} \cos \theta+\tilde{\mathcal{N}}^{r} \\
2 \rho^{\prime}+w^{\prime}-\left[\sum_{1}^{\infty}\left(-w_{n}^{2} \cos n \theta+w_{n}^{1} \sin n \theta\right)\right] & =\frac{1}{2} \sin \theta+\tilde{\mathcal{N}}^{\theta}
\end{aligned}
$$

where $\tilde{\mathcal{N}}^{r}$ and $\tilde{\mathcal{N}}^{\theta}$ are $o_{X}\left(\epsilon^{0}\right)$ and $\tilde{\mathcal{N}}_{0}^{r}=0$.

As discussed in [11] we can assume

$$
\tilde{\mathcal{N}}_{0}^{\theta}=0,\left(\tilde{\mathcal{N}}^{\theta}\right)_{1}^{j}=0, j=1,2
$$


and rewrite (4.7) in the form

$$
\begin{array}{r}
\sum_{2}^{\infty}\left(-n^{2}+n-2\right)\left(\rho_{n}^{1} \cos n \theta+\rho_{n}^{2} \sin n \theta\right)-2 \sum_{1}^{\infty}\left(w_{n}^{1} \cos n \theta+w_{n}^{2} \sin n \theta\right) \\
=\frac{1}{2} \cos \theta+\tilde{\mathcal{N}}^{r},( \\
\sum_{2}^{\infty} 2 n\left(\rho_{n}^{2} \cos n \theta-\rho_{n}^{1} \sin n \theta\right)+\sum_{2}^{\infty} 2 n\left(w_{n}^{2} \cos n \theta-w_{n}^{1} \sin n \theta\right)=\tilde{\mathcal{N}}^{\theta}
\end{array}
$$

where we have also used the Fourier series of $\rho, w, \rho^{\prime}, w^{\prime}, \rho^{\prime \prime}$. In $(4.8)_{2}$ we have set $w_{1}^{1}=-\frac{1}{4}$ that follows from $(4.8)_{1}$.

Lemma 4.1. Let $L(\rho, w)$ the l.h.s. of (4.8). Then

(i): $L(\rho, w) \in L^{2} \times L^{2}$.

(ii): The map $L: W^{2,2} \times W^{1,2} \rightarrow L^{2} \times L^{2}$ defined by

$$
W^{2,2} \times W^{1,2} \ni(\rho, w) \rightarrow L(\rho, w) \in L^{2} \times L^{2},
$$

is linear and bounded and has a bounded inverse $L^{-1}: L^{2} \times L^{2} \rightarrow$ $W^{2,2} \times W^{1,2}$.

Proof. By inspecting (4.8) and by observing that $(\rho, w) \in W^{2,2} \times W^{1,2}$ implies that the series in (4.8) are well defined $L^{2}$ functions proves (i) and also that $L$ is bounded. To show that $L^{-1}$ exists and is bounded we note that from (4.8) the equation

$$
L(\rho, w)=(p, v) \in L^{2} \times L^{2},
$$

is equivalent to the system

$$
\begin{gathered}
\left\{\begin{array}{c}
w_{1}^{1}=p_{1}^{1}, \\
w_{1}^{2}=p_{1}^{2},
\end{array}\right. \\
\left\{\begin{array}{l}
\left(2-n+n^{2}\right) \rho_{n}^{1}+2 w_{n}^{1}=-p_{n}^{1}, \quad, n \geq 2, \\
\left(2-n+n^{2}\right) \rho_{n}^{2}+2 w_{n}^{2}=-p_{n}^{2},
\end{array}\right. \\
\left\{\begin{array}{l}
2 n \rho_{n}^{2}+(n+1) w_{n}^{2}=v_{n}^{1}, \\
2 n \rho_{n}^{1}+(n+1) w_{n}^{1}=v_{n}^{2},
\end{array}, n \geq 2,\right.
\end{gathered}
$$


where (4.11) and (4.12) follow from (4.7) 1 and (4.13) from (4.7) 2 . Equations (4.12) and (4.13) imply

$$
\begin{aligned}
& \left(\begin{array}{l}
\rho_{n}^{1} \\
w_{n}^{1}
\end{array}\right)=\frac{1}{2-3 n+n^{3}}\left(\begin{array}{lr}
n+1 & -2 \\
-2 n & 2-n+n^{2}
\end{array}\right)\left(\begin{array}{c}
-p_{n}^{1} \\
v_{n}^{2}
\end{array}\right), \\
& \left(\begin{array}{l}
\rho_{n}^{2} \\
w_{n}^{2}
\end{array}\right)=\frac{1}{2-3 n+n^{3}}\left(\begin{array}{lr}
n+1 & -2 \\
-2 n & 2-n+n^{2}
\end{array}\right)\left(\begin{array}{l}
-p_{n}^{2} \\
v_{n}^{1}
\end{array}\right),
\end{aligned}
$$

and therefore

$$
\left\{\begin{array}{l}
\left|\rho_{n}^{j}\right| \leq \frac{C}{n^{2}} \sum_{i=1,2}\left(\left|p_{n}^{i}\right|+\frac{1}{n}\left|v_{n}^{i}\right|\right), \\
\left|w_{n}^{j}\right| \leq \frac{C}{n} \sum_{i=1,2}\left(\frac{1}{n}\left|p_{n}^{i}\right|+\left|v_{n}^{i}\right|\right),
\end{array}, \quad n \geq 2 .\right.
$$

These inequalities and (4.11) show that the Fourier coefficients $\rho_{n}^{j}, j=$ 1,$2 ; n=2, \cdots$ and $w_{n}^{j}, j=1,2 ; n=1, \cdots$ define functions $\rho \in W^{2,2}$ and $w \in W^{1,2}$ that satisfy (4.1).

From Lemma 4.1 it follows that (4.8) is equivalent to the equation

$$
(\rho, w)=(0, \bar{w})+L^{-1}\left(\begin{array}{c}
\tilde{\mathcal{N}}^{r}(\rho, w) \\
\tilde{\mathcal{N}}^{\theta}(\rho, w)
\end{array}\right):=G(\rho, w),
$$

where we have set $\bar{w}=-\frac{1}{4} \cos \theta$. Therefore the problem of solving (4.8) is reduced to the existence of a fixed point for the map $G: X \rightarrow X$.

Fix $M=2\|(0, \bar{w})\|$ in $(3.2)$. Then from the fact that $\tilde{\mathcal{N}}^{r}$ and $\tilde{\mathcal{N}}^{\theta}$ are $o_{X}\left(\varepsilon^{0}\right)$ and from Lemma 4.1 we have:

$$
\begin{aligned}
(\rho, w) \in X \Rightarrow\|G(\rho, w)\| & \leq\|(0, \bar{w})\|+C(\varepsilon), \\
\|G(\rho, w)-G(\tilde{\rho}, \tilde{w})\| & \leq C(\varepsilon)\|(\rho, w)-(\tilde{\rho}, \tilde{w})\| .
\end{aligned}
$$

Therefore, for $\varepsilon>0$ smaller than some $\varepsilon_{0}>0, G: X \rightarrow X$ is a contraction and (4.16) has a unique solution $\left(\rho^{*}, w^{*}\right) \in X$. Due to the equivalence between (4.16) and (4.8), $\left(\rho^{*}, w^{*}\right)$ is a solution of (2.7). Moreover (4.17) imply:

$$
\begin{aligned}
\left\|\rho^{*}\right\|_{W^{2,2}} & \leq C(\varepsilon), \\
\left\|w^{*}-\bar{w}\right\|_{W^{1,2}} & \leq C(\varepsilon),
\end{aligned}
$$

where $C(\varepsilon) \rightarrow 0$ as $\varepsilon \rightarrow 0^{+}$. From these estimates and Lemma 3.1 it follows that the r.h.s. of system $(2.7)$ computed for $(\rho, w)=\left(\rho^{*}, w^{*}\right)$ is of class $C^{0, \gamma}$. Therefore we can regard $(2.7)$ as a system of o.d.e. with a Hölder continuous r.h.s. This shows that $\rho^{*} \in C^{2, \gamma}$ and $w^{*} \in C^{1, \gamma}$. The uniqueness in (iii) is just a restatement of the fact that $\left(\rho^{*}, w^{*}\right)$ is the unique fixed point of a contraction on $X$. The properties of $s$ in (iv) follow (1.10) and from 
(i) and (ii) recalling also the expression of $c(\epsilon)$ and (4.6). The statement on the smoothness of $\Omega$ follows from (2.6) $)_{2}$ and (i) and (ii). The expression

of $\bar{\Omega}$ follows from $(2.6)_{2}$ and from the computation of $\left\langle\frac{f}{\omega}\right\rangle$ (see [11]). The proof of Theorem 1.1 is concluded.

\section{Acknowledgment}

It is a pleasure for G. Fusco and P. Negrini to thank the CAMGSD, Department of Mathematics at IST for the kind hospitality during the preparation of the present work.

\section{References}

[1] I. Newton. Philosophiae naturalis principia mathematica. Londini: Jussi Societatus Regiae ac typis Joseph Streater, 1687.

[2] Chandrasekhar, S. Ellipsoidal figures of equilibrium. New York: Dover, 1987.

[3] W. M. Oliva. Massas Fluidas em Rotação e os Elipsóides de Riemann - uma Abordagem Informal. Rev. Matemática Universitária 43 (2007), pp. 28-37.

[4] B. Riemann. Ein beitrag zu den unterschungen über die bewengung eines flüssigen gleichartigen ellipsoides. Abhandlungen der Königlichen Gesellschaft der Wissenschaften zu Göttingen 9 (1860), pp. 3-36.

[5] J. P. G. L. Dirichlet. Untersuchungen über ein problem der hydrodynamik. Aus dessen nachlass hergestellt von R. Dedekind. Abhandlungen der Königlichen Gesellschaft der Wissenschaften zu Göttingen 8, (1858/1859) pp. 225-264.

[6] H. Poincaré. Sur l'équilibre d'une masse fluide animée d'un mouvement de rotation. Acta mathematica 7 (1885), pp. 259-380.

[7] S. Kowalewsky. Zusätze und Bemerkungen zu Laplace's Untersuchung über die Gestalt der Saturnsringe. Astronomiche Nachrichten (1885), pp. 38-48.

[8] J. F. G. Auchmuty. Existence of axisymmetric equilibrium figures. Arch. Rat. Mech. Analysis 65 (1977), pp. 249-261.

[9] J. F. G. Auchmuty and R. Beals. Variational solutions of some nonlinear freeboundary problems. Arch. Rat. Mech. Analysis 43 (1971), pp. 255-271.

[10] L. A. Caffarelli and A. Friedman. The shape of axisymmetric rotating fluid. J. Funct. Analysis 35 (1980), pp. 109-142.

[11] G. Fusco, P. Negrini and W. M. Oliva. Stationary motion of a thin self-gravitating toroidal incompressible liquid layer. Preprint (2011).

[12] C. Miranda. Sulle proprietá di regolaritá di certe trasformazioni integrali. Mem. Accademia dei Lincei VII, Sez.I ${ }^{a}$ (1965), pp. 303-336. 